according to Nagel, were not as good at herding their females as were the pure hamadryas. This reduction in the fitness of the hybrid males might be sufficient to account for the narrowness of the hybrid zone, for which it can be argued theoretically that only a relatively small reduction in the fitness of the hybrids is needed (Slatkin, Genetics, in the press). Because anubis and hybrid males had the same opportunities to learn the herding behaviour as the pure hamadryas, Nagel concludes that the tendency to herd females and form permanent harems is at least in part genetically determined.

Another salient factor, in Nagel's view, is the desirability of small foraging units in the habitat occupied by the hybrid population. Even in the pure anubis group, there was the tendency to break up into foraging groups which would be apart for some part of the day and occasionally sleep apart. The anubis social system was not flexible enough, however, to take full advantage of the small foraging groups and the anubis range was thus limited by the inability to displace the hamadryas from the arid region. This failure to adapt once more suggests a genetic component. On the other hand, the anubis tendency to sleep in trees and the hamadryas tendency to sleep in rocks seemed to have no genetic component, each species apparently utilising the most accessible and the safest (from predators) sleeping sites available.

The existence of the hybrid zone incidentally raises questions about the classification of the genus Papio. The situation in the taxonomy of primates is already so complex that the common names of the primates are frequently more useful than the scientific names. Although five species of African baboons have been recognised ( $P$. hamadryas, $P$. anubis, $P$. cynocephalus, $P$. papio and $P$. ursisus) there have been observations of free hybridisation between $P$. cynocephalus and $P$. anubis in Kenya (Maples, Am. J. phys. Anthorp., 36; 1972) and now between the anubis and the hamadryas in at least two locations in Ethiopia. The conditions begin to argue for reclassification of Papio as a single species or a 'super-species' with different geographical races. Unless emotional attachment to the labelling of fellow primates prevents it, there will have to be yet another change in the nomenclature. Certainly greater differences in morphology of geographic races exist in other species.

M.S.

\section{New method of predicting earthquakes}

from our Geomagnetism Correspondent

AFTER many years of pessimism in which limited progress towards a solution seemed hardly to justify the vast effort of data collection, earthquake prediction studies seem to have entered a new, more hopeful phase. Although it is less than a year since Whitcomb et al. (Science, 180, 632; 1973) reported their discovery of $V_{\mathrm{p}} / V_{\mathrm{s}}$ ratio changes preceding the San Fernando earthquake of 1971 and proposed a physical model to explain them, Gupta (Science, 182, 1129 ; 1973) has come up with a quite different premonitory phenomenon which involves seismic velocities. As a method of predicting earthquakes it seems to be potentially as useful as the earlier discovery and can be explained in terms of the same physical model, but it has the additional practical advantage that it can be applied using a single three-component seismograph.

The phenomenon itself involves the splitting of $\mathrm{S}$ waves and is not, as such, a new discovery. Both laboratory and theoretical studies (see, for example, Nur and Simmons, J. geophys. Res., 74, 6667 ; 1969) have shown that when nonhydrostatic stress is applied to a rock containing cracks, an anisotropy is induced in the $\mathrm{S}$ wave velocity causing the $\mathrm{S}$ wave to split into two distinct components with different velocities. By analogy with double refraction behaviour of light waves in stressed glass, the seismic version has come to be known as "acoustic double refraction" and its magnitude, expressed as the difference between the two component velocities, increases as the deviatoric stress increases.

The question now arises, of course, as to whether the phenomenon investigated theoretically and found experimentally in the laboratory can be observed in the field. Gupta has demonstrated that it can, at least in central Nevada where the faulting is primarily related to crustal extension. The main events for which Gupta sought premonitory (and subsequent) $\mathrm{S}$ wave splitting were two earthquakes of magnitude 4.0 and 3.9 with epicentres in the Slate Mountain and Mina regions, respectively; and the sources of the observed $\mathrm{S}$ waves were the foreshocks and aftershocks of these events. For each main event the difference $(\Delta t)$ in the arrival times between the two $\mathrm{S}$ wave components gradually increased to a maximum and then decreased again towards its original value; and in each case the main shock took place just after the peak $\Delta t$ had been reached. In numerical terms, the peak values of $\Delta t$ lay in the range $0.7-0.9 \mathrm{~s}$, which compares with the apparently normal value of about $0.2 \mathrm{~s}$. The peak $\mathrm{S}$ wave splitting was thus small (about $2.5 \%$ of the total travel time). On the other hand, it was just about the greatest likely to be observed at this location because $\mathrm{S}$ wave velocity anisotropy is greatest along the direction of crustal extension and the source-receiver line was in each case within about $20^{\circ}$ of this direction.

On observational grounds alone, therefore, frequent monitoring of $\Delta t$ values would seem to be a promising prediction technique-and a particularly attractive one in that, in principle, a given area can be monitored using just one three-component seismograph. On a theoretical level, Gupta claims that the observed $\Delta t$ variation is consistent with pre-earthquake stress accumulation according to the elastic rebound theory, and he has already (Bull. seism. Soc. $A m ., 63,1157$; 1973) shown it to be explicable in terms of the dilatancyfluid saturation model used to explain premonitory $V_{\mathrm{p}} / V_{\mathrm{s}}$ changes. The time scales of the two precursors are also apparently comparable. Whitcomb et al. found, for example, that the precursor time interval for a magnitude 4.0 event is about 25 days. For Gupta's magnitude 4.0 shock $\Delta t$ began to rise from its "normal" value of $0.2 \mathrm{~s}$ just over 30 days before the shock.

\section{Helical \\ hypodermic}

\section{from a Correspondent}

BACTERIOPHAGE, literally 'bacteria eater', is the term used to describe viruses which attack bacteria. Some of these are very elaborate, for example, $\mathrm{T}$ phage, and have morphologically distinct head and tail assemblies. The phage fastens itself tail first onto the surface of its victim, injects its genetic material through the tail into the interior of the bacterium, and thereby assumes the biochemical machinery of the host and rapidly spawns new phage progeny. The key process of infection is therefore the penetration of the bacterial cell wall by the phage tail. It is powered by a contraction of the phage tail sheath from a long narrow tubular structure to a short fat one. Since the sheath is a relatively simple structure comprising a regular helical arrangement of protein subunits, it provides an excellent system for the study of an elementary contractile process which is not only of intrinsic interest but which may also serve as a useful model in the analysis of contraction in more advanced organisms. In one important respect, however, the sheath's activity differs from that of other contractile systems, such as flagella and muscle, since the contraction occurs once and for all, that is, it is not reversible.

In the case of $T$-even phages, the presence of sheaths in the extended 